\title{
Observations, Perceptions Et Adaptations Au Changement Climatique Dans La Sous-Préfecture De Séguéla (Côte D’ivoire) \\ ${ }^{1}$ YAO-KOUASSI Quonan Christian, ${ }^{2}$ ADAYÉ Kouassi Albert, ${ }^{3}$ KOUADIO Konan Célestin, ${ }^{4}$ COULIBALY Yardjouma Esai
}

\author{
${ }^{1}$ Maître-assistant \\ Université Jean Lorougnon Guédé (Daloa, Côte d'Ivoire) \\ ${ }^{2}$ Enseignant-chercheur \\ Université Jean Lorougnon Guédé (Daloa, Côte d'Ivoire) \\ ${ }^{3}$ Enseignant-chercheur \\ Université Jean Lorougnon Guédé (Daloa, Côte d'Ivoire) \\ ${ }^{4}$ Université Jean Lorougnon Guédé (Daloa, Côte d'Ivoire)
}

\begin{abstract}
In a context of sustainable development and food security, the fight against climate change has become a priority. In tropical rural areas, its effects are beginning to appear by affecting agricultural production, which is a factor that aggravates socio-economic impacts. Indeed, understanding the determinisms of climate change as well as their perception and the adaptation strategies developed by rural populations is an issue of food security and socio-economic development. In the sub-prefecture of Séguéla, the perceptions and adaptations to climate change of populations living from rain-fed agriculture are acute. This article analyzes on the one hand the climatic variability of this sub-prefecture based on scientific observations of the annual accumulations of precipitation from 1986 to 2015. And on the other hand, following a questionnaire survey, the perception and representation of this climatic variability as well as the strategies developed in response by farmers were noted. The main results indicate a decrease in rainfall in general modifying the crop calendar of farmers. In addition, the study attests that peasant perceptions and representations are linked to their local beliefs. This then results in dynamics and modalities of adaptation through the introduction of new cultures / associations of cultures, the development of lowlands reflecting the interactions of the peasant population with its environment.
\end{abstract}

Key words : Climate change, Observations, Perception, Adaptation, Sub-prefecture of Séguéla

\section{Résumé}

Dans un contexte, de développement durable et de sécurité alimentaire, la lutte contre le changement climatique est devenue une priorité. Dans les zones rurales tropicales, ses effets commencent à se manifester en affectant les productions agricoles constituant un facteur aggravant des impacts socio-économiques. En effet, comprendre les déterminismes du changement climatique ainsi que leur perception et les stratégies d'adaptation développées par les populations rurales est un enjeu de sécurité alimentaire et de développement socio-économique. Dans la sous-préfecture de Séguéla, les perceptions et adaptations au changement climatique des populations vivant d'agriculture pluviale se posent avec acuité. Cet article analyse d'une part la variabilité climatique de cette sous-préfecture en s'appuyant sur des observations scientifiques des cumuls annuels des précipitations de 1986 à 2015. Et d'autre part, à la suite d'une enquête par questionnaire, le ressenti et la représentation de cette variabilité climatique ainsi que les stratégies développées en réponse par les exploitants agricoles ont été relevés. Les principaux résultats indiquent une baisse de la pluviométrie de manière générale modifiant le calendrier cultural des exploitants agricoles. En outre, l'étude atteste que les perceptions et représentations paysannes sont en lien avec leur croyance locale. Il en résulte alors des dynamiques et des modalités d'adaptation à travers l'introduction de nouvelles cultures/associations de cultures, la mise en valeur des bas-fonds traduisant les interactions de la population paysanne avec son environnement. 
Mots clés : Changement climatique, Observations, Perception, Adaptation, Sous-préfecture de Séguéla

\section{Introduction}

Les observatoires territorialisés de pratiques agricoles développent une réflexion sur l'observation des pratiques à une échelle spatiale adaptée sans qu'il ne soit fait explicitement état, pour l'instant, de la prise en compte du changement climatique (Ahmadi et al., 2013, p.62). En milieu rural ouest-africain particulièrement, où l'adaptation est davantage susceptible d'être autonome, l'étude des perceptions représente un intérêt majeur. Il est en effet acquis que les facteurs cognitifs, via la perception des changements, la perception des risques et la perception des capacités d'adaptation, jouent un rôle dans le processus d'adaptation (Hansen et al., 2004, p.2). On n'a d'une part la connaissance climatologique fournit par les spécialistes/chercheurs et d'autre part le changement climatique qui lui est perçu à l'échelle territoriale ou locale, voire personnelle à partir de situation inusitée en fonction de ses habitudes, attentes et croyances. De plus dans le cadre du changement climatique en cours, le régime de pluie en Afrique de l'ouest fait partie de ceux comportant plus d'incertitudes (Deme et al., 2016, p.61). À l'échelle de la Côte d'Ivoire avec une population rurale, les perturbations des régimes pluviométriques modifient les bénéfices tirés de l'activité agricole. Ainsi, l'interrogation sur les raisons de l'évolution de ces derniers et les innovations rurales qui en découlent, demeurent intactes. Dans la région du Worodougou, la sous-préfecture de Séguéla a une économie essentiellement basée sur l'agriculture. Les paysans se consacrent totalement à l'agriculture vivrière pluviale dominée par la culture du riz, du maïs et de l'igname. Cette dépendance à l'agriculture pluviale la rend vulnérables aux variabilités climatiques. Ainsi, on observe une récession pluviométrique dans la région qui s'illustre par une perturbation du calendrier cultural avec des conséquences sur la sécurité alimentaire. En nous appuyant sur différentes analyses d'observations, nous nous proposons d'interroger les perceptions paysannes des changements climatiques des populations de Séguéla. Nous tenterons également de comprendre comment ces mêmes populations parviennent-elles à mettre en place une gestion adaptative de leurs exploitations agricoles.

\section{Présentation de la zone d'étude}

La sous-préfecture de Séguéla est située dans la région du Worodougou au nord-ouest de la Côte d'Ivoire (figure 1). Elle possède un climat de savane avec une pluviométrie moyenne de $1182 \mathrm{~mm} / \mathrm{an}$. La zone d'étude a un relief essentiellement constitué de plateaux et de collines granitiques avec une altitude moyenne de $260 \mathrm{~m}$. Elle couvre 54 villages, soit environ 63774 habitants en 2014, sur une superficie de 842,15 $\mathrm{Km}^{2}$. Les paysans de cette sous-préfecture pratiquent l'agriculture pluviale et l'élevage, qui représentent la première et la deuxième source de revenus. Les exploitations agricoles de cultures pluviales sont dominées par le maïs, l'igname et le riz. Ces dernières sont généralement sur des sols sablonneux, constitués de granite et de micas.

\section{$\underline{\text { Figure } 1 \text { : Situation de la zone d'étude }}$}

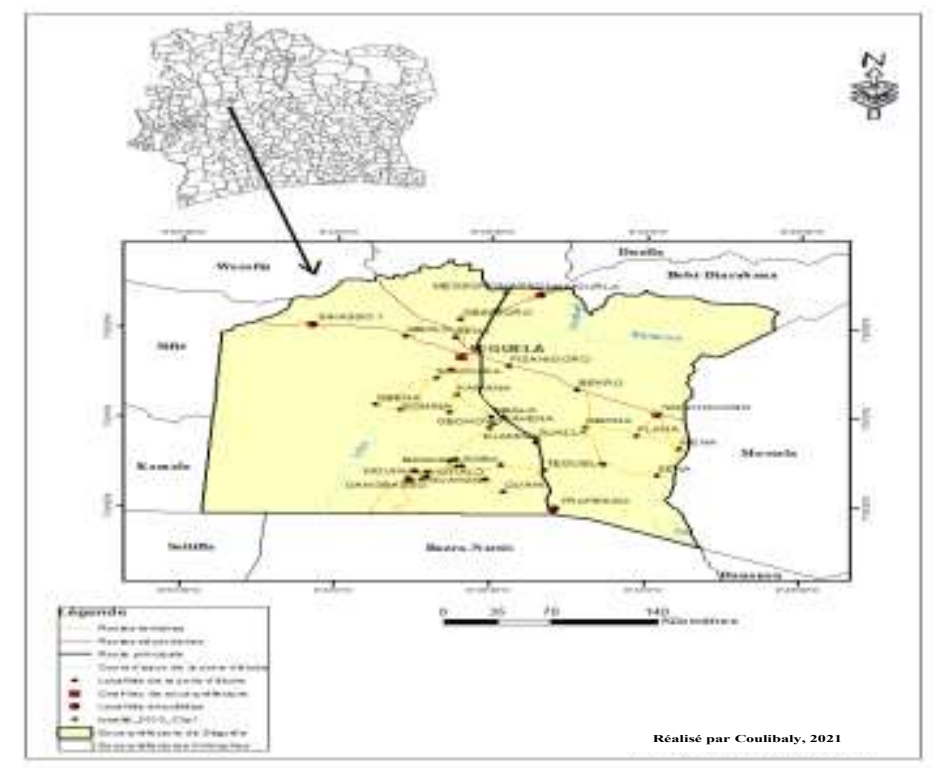




\section{Données et méthodes}

Cette étude s'appuie sur une méthodologie quantitative et qualitative. L'approche quantitative va du recueil des données des observations scientifiques du climat de la sous-préfecture de Séguéla à celui des perceptions anciennes et nouvelles du climat par les exploitants agricoles à travers un questionnaire. Ces dernières sont ensuite confrontées aux données climatiques (températures et pluie) obtenues. En revanche, l'approche qualitative à travers l'enquête par questionnaire auprès des villages a servi à identifier les logiques sociales d'adaptation des pratiques agricoles aux changements climatiques.

Les données utilisées sont celles de la SODEXAM (Société d'exploitation et de développement aéroportuaire, aéronautique et météorologique) couplées à celle du MINADER (Ministère de l'agriculture et du développement rural). En effet, celles de la SODEXAM couvrent seulement la période de 1986 à 2015, tandis que les données obtenues du MINADER portent sur la période de 2010 à 2020. Les différents jeux de données issues de relevées mensuelles portent sur les pluies. Les séquences sèches et humides durant la période 1986-2015, de même que l'évolution de la pluviométrie sont ainsi mises en lumière et le lien entre pluviométrie, production et rendement agricole établi.

Afin de recueillir la diversité des perceptions du climat et la multiplicité des pratiques adaptatives des paysans et les confronter aux jeux de données scientifiques, nous avons mené une enquête. Les enquêtes ont été réalisées sur 5 villages représentatifs de la zone agricole de notre terrain d'étude selon la méthode non probabiliste. Ainsi, les critères retenus sont l'âge des agriculteurs, ces derniers devaient avoir au moins 45 ans et une ancienneté dans l'agriculture d'au moins 10 ans. Dès lors, nous avons interrogé 170 exploitants agricoles à raison de 34 par village. Le questionnaire soumis à chaque exploitant agricole a permis de reconstituer le type d'activité agricole effectué au cours des dix dernières années. Il porte en outre sur les caractéristiques socioculturelles, les cultures vivrières pratiquées, la perception du climat actuel, des niveaux des calendriers et d'évolution des pluies. Enfin, les exploitants agricoles ont été interrogés sur les stratégies d'adaptation mobilisées pour améliorer la sécurité alimentaire face aux changements de température et de la pluviosité ressentis. L'ensemble des données recueillies ont été codées puis traitées à l'aide du logiciel Excel ; le logiciel khronostat 1.01 a servi quant à lui à l'analyse des données pluviométriques.

\section{Résultats}

\subsection{Changements climatiques observés dans la sous-préfecture de Séguéla}

L'évolution décennale des cumuls annuels des précipitations est l'indicateur le plus utilisé pour montrer l'évolution à long terme des pluies. Elle permet de mettre en évidence les années supérieures et inférieures à la moyenne climatologique et de décliner à partir de la variabilité interannuelle les grandes tendances de l'évolution des pluies ressentie. Dans la sous-préfecture de Séguéla, les pluies ont connu une alternance très forte entre 1986 et 2015 comme en témoigne la figure 2. Cette alternance est marquée par deux saisons sèches entre les périodes 1986-1993 et 2001-2009 ainsi que deux saisons humides entre 1994-2000 et 20102015.

Figure 2 : Évolution des précipitations moyennes annuelles observées de 1986 à 2015

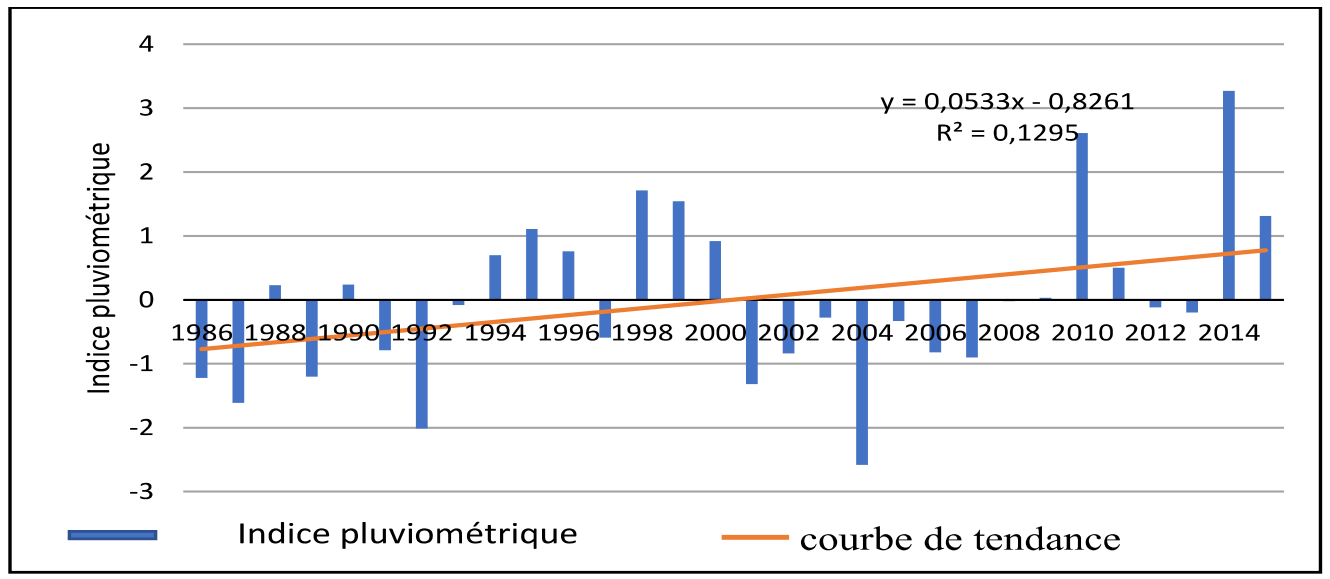

Source : Sodexam, 2016 
La première séquence sèche commence à partir de 1986 et s'achève en 1993 soit 8 ans de sècheresse avec une petite humidité pendant les années 1988 et 1990. Cette séquence à une moyenne pluviométrique de 1082,6 mm largement inférieure à la moyenne de toute la série qui est de $1216 \mathrm{~mm}$. Cette sècheresse qui s'achève en 1993 est due à une reprise de la pluviométrie à partir de 1994 qui dure jusqu'en 2000. Ces 7 ans d'humidité moyenne sont marqués par une année de sècheresse en 1997 qui présente une moyenne de $1361,5 \mathrm{~mm}$. Suite à la phase progressive de pluie, nous avons une nouvelle séquence sèche qui dure 9 ans (2001-2008) avec une moyenne pluviométrique de 1085,9 mm. En revanche, si la pluviométrie a connu une reprise après cette longue séquence sèche, on observe une petite période humide de 6 ans de 2010 à 2015 avec une moyenne de $1419 \mathrm{~mm}$. En définitive, les données d'observation montrent que la variation interannuelle de la pluviométrie dans la sous-préfecture de Séguéla est marquée par 17 ans de sècheresse sur 30 années.

L'analyse des observations du cumul pluviométrique annuel à elle seule, ne démontre pas totalement la réalité à laquelle les exploitants agricoles de la sous-préfecture de Séguéla sont confrontés. En effet, les observations les plus importantes pour ces exploitants agricoles restent celles du cumul pluviométrique mensuel (figure 3). On note que la sous-préfecture de Séguéla qui a un régime pluviométrique unimodal, enregistre des quantités importantes de pluie de février à septembre avec 5259,4 mm, ensuite, s'ensuit des mois secs d'octobre à janvier.

Figure 3 : Évolution inter-mensuelle de la pluviométrie de 1986 à 2015

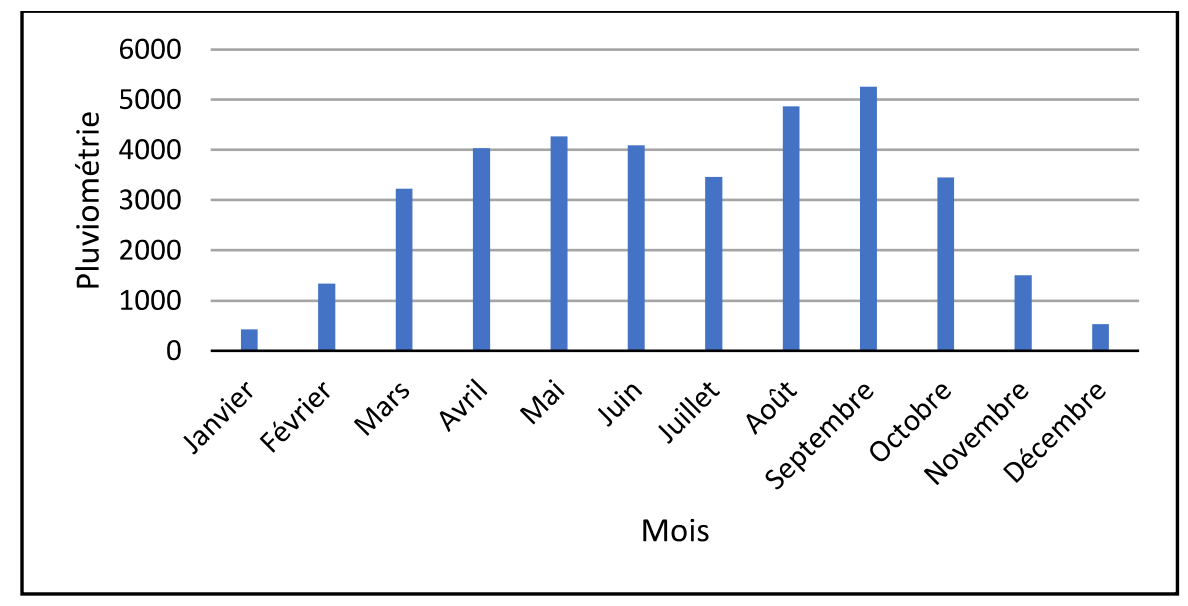

Source : Sodexam, 2016

\subsection{Perception des exploitants agricoles de la sous-préfecture de Séguéla au changement climatique}

Les exploitants agricoles de Séguéla sont les premiers acteurs à observer et subir les modifications du climat. Ainsi, les données sociales de leur perception de tout changement est un indicateur intéressant tant sur le plan climatique que les stratégies d'adaptation mise en œuvre. Nos résultats montrent que $90 \%$ des exploitants agricoles échantillonnés déclarent être confrontés à une forme du changement climatique due à des variations intermensuelles très prononcées de la pluie qui perturbent l'année agricole. De manière générale (Figure 4), les exploitants agricoles perçoivent également un début tardif des précipitations (85\%) associé à une fin précoce (95\%). Cependant, une part faible des exploitants agricoles (15\%) observe un début de saison précoce avec 5\% qui considèrent tardif la fin de la saison pluvieuse.

Figure 4 : Début et fin des saisons des pluies selon les agriculteurs 


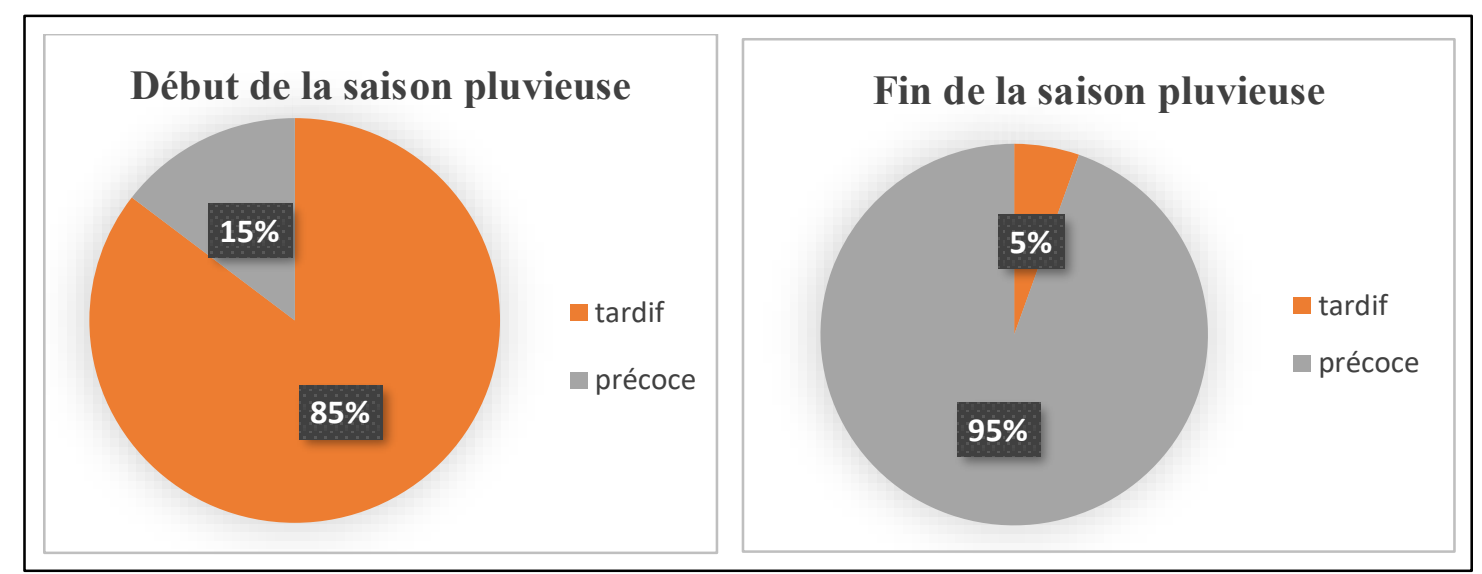

Source : Nos enquêtes, 2021

Nos résultats (figure 5) de nos enquêtes montrent également que la perception de la durée des précipitations diffère selon les exploitants agricoles. Ainsi, selon les enquêtés par le passé, la durée de la saison pluvieuse était de 8 mois (57\%) pour certains alors que 32\% et 11\% l'estiment respectivement à 7 mois et 5 mois. Par contre, la durée de la saison pluvieuse est perçue par 50\% des enquêtés à 5 mois aujourd'hui (50\%), tandis que $38 \%$ la soulignent à 4 mois et $12 \%$ à 3 mois.

Figure 5 : Durée passée et actuelle de la saison pluvieuse selon les agriculteurs

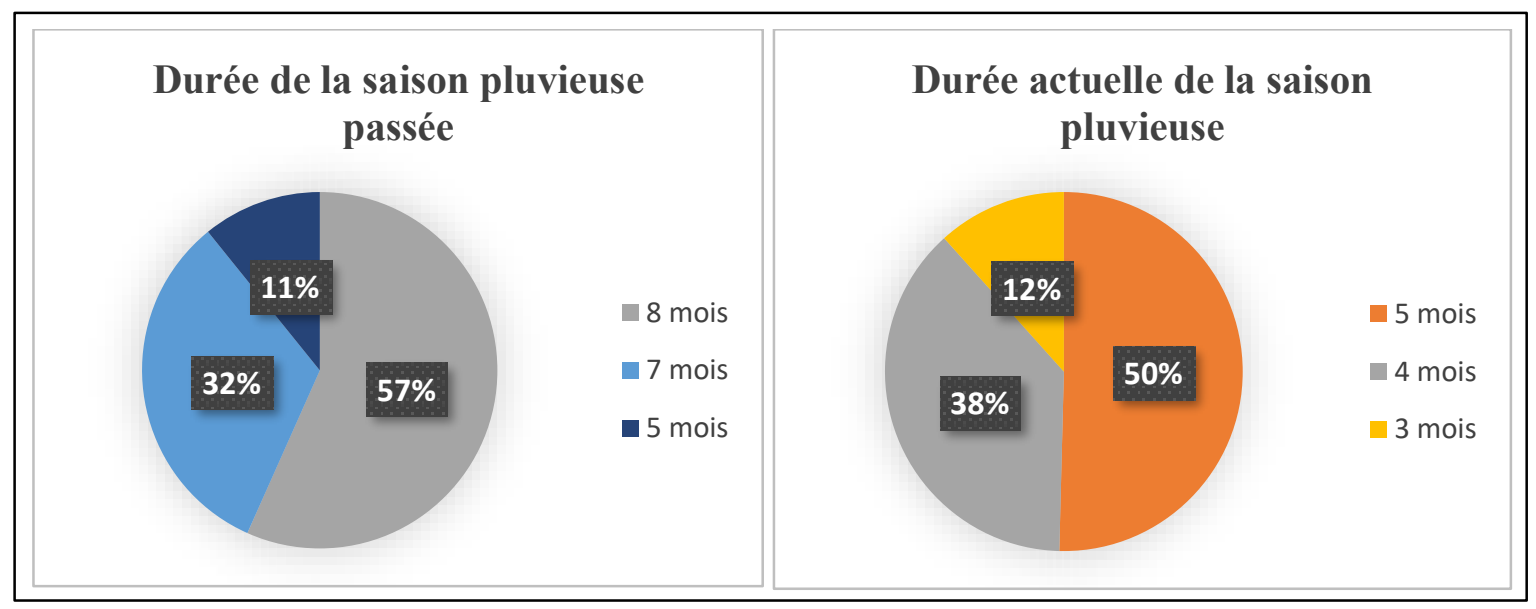

Source : Nos enquêtes, 2021

La dissemblance de ces résultats atteste de la difficulté des exploitants agricoles à évaluer l'évolution des précipitations. Les perceptions du décalage du début ou de la fin de la saison pluvieuse ainsi que la durée par les agriculteurs convergent avec les observations scientifiques.

\subsection{Quelles innovations et pratiques adaptives des exploitants agricoles de la sous-préfecture de Séguéla face au changement climatique ?}

Les stratégies d'innovation et d'adaptation mobilisées par les agriculteurs de la sous-préfecture de Séguéla sont des réponses au changement climatique observé. Ainsi, la date des semis des cultures qui est capitale a été changée par $90 \%$ des agriculteurs. De ce fait, ils ont adapté les dates de leurs semailles en débutant à partir de la fin avril pour finir à la fin juin. Cependant, peu d'exploitants agricoles (10\%) s'aventurent à changer la période des semis mais pratiquent plutôt des semis multiples. En outre, $90 \%$ des exploitants agricoles affirment introduire de nouvelle culture (le manioc est la culture la plus associée car moins exigeante en eau) en plus de l'expansion des espaces cultivés. Cette dernière s'accompagne très souvent de polyculture et de mise en valeur des basfonds. Ainsi, nos résultats montrent que $70,6 \%$ des agriculteurs pratiquent la polyculture contre $29,4 \%$ qui font de la monoculture. 
Figure 6: Modification du temps des semis des agriculteurs de la sous-préfecture de Séguéla

\begin{tabular}{|c|c|c|c|c|c|c|c|c|c|c|c|c|}
\hline \multirow[b]{2}{*}{ Activités } & \multicolumn{12}{|c|}{ Ancien calendrier agricole } \\
\hline & Jan & Fév & Mars & Avril & Mai & Juin & Juillet & Août & Sept & Oct & Nov & Déc \\
\hline \multicolumn{13}{|l|}{ Défrichage } \\
\hline \multicolumn{13}{|l|}{ Labour } \\
\hline \multicolumn{13}{|l|}{ Semis } \\
\hline \multicolumn{13}{|l|}{ Récolte } \\
\hline \multicolumn{13}{|c|}{ Nouveau calendrier agricole } \\
\hline \multicolumn{13}{|l|}{ Défrichage } \\
\hline \multicolumn{13}{|l|}{ Labour } \\
\hline \multicolumn{13}{|l|}{ Semis } \\
\hline Récolte & & & & & & & & & & & & \\
\hline
\end{tabular}

Source : Nos enquêtes, 2021

Notons que cette polyculture ne se fait pas uniquement par l'association de culture vivrière (mais, mil, sorgho) mais également avec des cultures de rentes. Ce sont $52 \%$ des agriculteurs (figure 7) qui adoptent l'association des cultures de rentes/vivriers contre $48 \%$ des agriculteurs qui pratiquent l'association vivrier/vivriers.

Figure 7 : Proportion des différents types de polyculture selon l'enquête de terrain

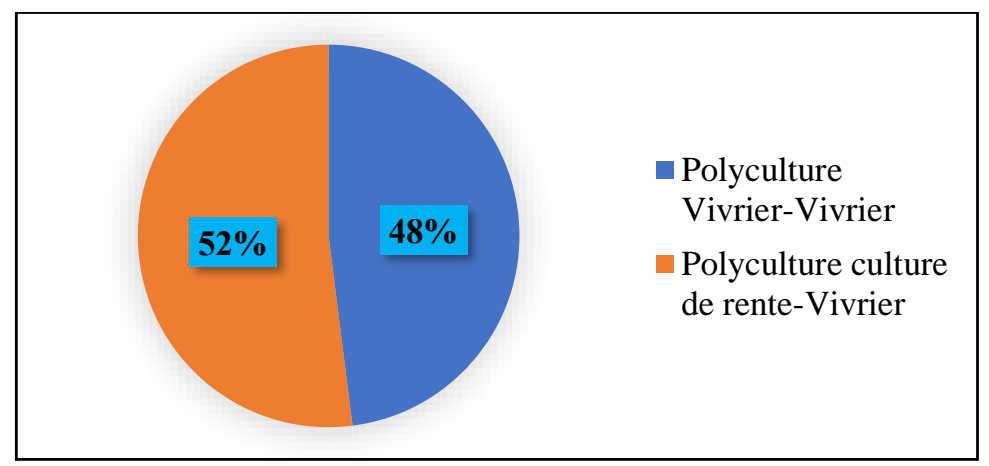

Source : Nos enquêtes, 2021

L'épandage voire l'usage des produits chimiques comme les engrais et les produits phytosanitaires joue un rôle prépondérant parmi les leviers d'adaptation mobilisés par les exploitants agricoles de la sous-préfecture de Séguéla. En effet, nos résultats (Figure 8) montrent que 60\% des enquêtés utilisent des engrais, contre $30 \%$ qui utilisent en plus des engrais des produits phytosanitaires. Seulement $10 \%$ des agriculteurs affirment ne pas avoir recours aux produits chimiques pas manque de moyen financier.

Figure 8 : Proportion des agriculteurs ayant recours aux produits chimiques

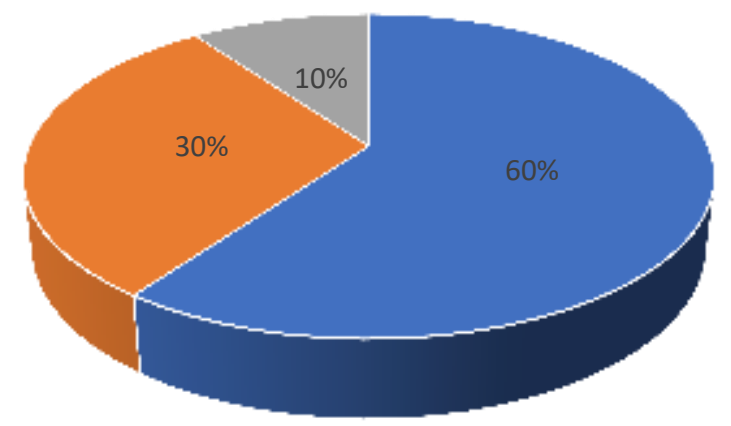

- Engrais - Engrais et produits phytosanitaires = Aucun produit utilisé

Source : Nos enquêtes, 2021 


\section{Discussion}

\subsection{Dynamique d'observations du climat}

Les rythmes climatiques saisonniers étant dictés par l'alternance entre saison des pluies et saison sèche, il est légitime de regarder si les changements climatiques ne se manifestent pas seulement en termes de volumes précipités mais aussi quand et comment les pluies commencent et s'arrêtent (Debreuil et al., 2021, p.256). En effet, dans la sous-préfecture de Séguéla l'allongement de la saison sèche ainsi que la distribution de la pluie à l'intérieur de cette sous-préfecture a pour conséquence la modification du calendrier cultural. Ainsi, les cultures du riz, du maïs et de l'igname, très exigeantes en eau ont des difficultés d'atteintes de la maturité démontrant alors le lien manifeste avec la variabilité climatique observée. Les travaux de Kosmowski et al., (2015, p.98) sur Bambey ont permis de confirmer des tendances significatives vers une diminution de la saison des pluies entre 1970 et la fin des années 1990. Ils montrent que la reprise apparaît tardivement puisque le cumul pluviométrique reste faible autour de $450 \mathrm{~mm} / \mathrm{an}$ puis augmente rapidement pour atteindre une moyenne de plus de $600 \mathrm{~mm} / \mathrm{an}$ au cours des dix dernières années, soit une augmentation de $50 \%$ en vingt ans. De plus, ils montrent qu'ils existent une corrélation significative entre le cumul pluviométrique et la date de démarrage des pluies qui traduit que $25 \%$ des variations de la pluviométrie annuelle sont expliqués par des fluctuations du démarrage de la saison des pluies. Pour ces auteurs, le choix de la date des semis est un élément crucial dans la stratégie de l'agriculteur qui doit s'assurer que le semi ne soit pas suivi d'une longue séquence sèche et que la plante arrive à maturation à la fin de la saison des pluies. Par ailleurs, L'Hôte et al., (2002, p.565) montrent que les déficits pluviométriques entre les périodes 1970- 2000 et 19601969 sont de $211 \mathrm{~mm}$, soit une baisse de 36,2 \% et ont confirmé la tendance générale à la baisse de la pluviométrie en Afrique de l'Ouest. Ces évolutions sont importantes car le déroulement des dates de début et de fin de la saison pluvieuse est un facteur majeur de contrôle des écosystèmes (phénologie, hydrologie) et impose aussi de fortes contraintes aux pratiques agricoles, la plupart des productions étant des cultures pluviales (Debreuil et al., 2021, p.258).

\subsection{Des formes de perceptions à la représentation du changement climatique}

En faisant un saut des modèles de la «perception » à ceux de la «représentation », on est à même de mieux comprendre la rationalité sociale et les ressources interprétatives communes qu'elle réfléchit et cultive. Alors que la perception est articulée à des savoirs sensoriels, la représentation est construite par rapport à des symboles, par rapport à la réalité sociale et par rapport à un savoir social (Joffe, 2005, p.124). Ainsi, les perceptions paysannes des changements climatiques et environnementaux s'expriment autour des représentations qu'ils se font des éléments météorologiques que sont les précipitations, les températures et les vents, et les changements qui affectent leur environnement naturel : mares, végétation, cours d'eau (Attané et $a l, 2015$, p.116). En effet, pour les exploitants agricoles de la sous-préfecture de Séguéla, la perturbation de la saison pluvieuse se manifeste par l'arrivée tardive de la floraison du karité et l'apparition des puces rouges qui indiquent la période de semence de l'arachide. Comme ailleurs en Afrique, l'imminence de l'une ou de l'autre saison de l'année peut se lire dans le comportement de la nature. Ainsi, il est toujours vérifié que lorsque le vent souffle de façon continue d'ouest en est, toute une journée, en fin de saison sèche, cela annonce l'arrivée des pluies les jours suivants (Brou et al., 2007, p.72). De même, la qualité de perception du climat revêt aussi de facteurs endogènes liés aux valeurs culturelles et sociales. Ainsi, les exploitants agricoles de Séguéla se représentent la réduction de la saison de pluie comme étant liée en partie à la colère de Dieux contre «les pratiques malsaines des hommes ». Pour la plupart des populations rurales, la pluie est un don de Dieu. Nombreux sont donc ceux qui s'imaginent que la diminution progressive de «l'eau venant du ciel » est liée au non-respect des valeurs ancestrales telles que la profanation des lieux sacrés, les relations sexuelles discrètes en brousses (Brou et al., 2007, p.74). L'expression de certains stimuli est si tenue que leur perception relève presque du subconscient. Bien souvent, ces sociétés médiatisent l'occurrence de ses signaux à travers le filtre de croyances animistes mettant en scène des forces supranaturelles (Dounia et $a l, 2013$, p.181). Des résultats similaires sont obtenus par Attané et $a l$, (2015, p.122), qui dans leurs travaux sur la région de Bondoukou au Niger, confirment que les paysans considèrent que l'abandon des rites pratiqués par les zimmas détenteur des croyances animistes locales au profit de nouvelles pratiques islamiques interprétées comme un grand péché serait à l'origine de cette situation de changements climatiques et environnementaux rendant les saisons et les productions agricoles imprévisibles et précaires. Ces interprétations se construisent aujourd'hui autant par le système de 
croyances locales que par les connaissances scientifiques auxquelles les paysans africains ont accès (Kosmowski et al., 2015, p.108).

\subsection{Vers des réponses adaptatives variées pour une résilience au changement climatique}

L'adaptation des pratiques culturales nécessite de mieux comprendre la réponse des arbres aux changements environnementaux (Laclau et al.,2015, p.190). En effet, l'adaptation n'est pas uniquement une nécessité objectivée par les expertises internationales et imposées par les conditions naturelles extérieures, elle est aussi le choix des individus et des communautés, construit à partir des connaissances scientifiques et des expériences collectives des dangers, des dispositions (normes et valeurs individuelles et collectives) qui modèlent les façons de penser et d'agir, des coûts économiques, socioculturels et symboliques de l'adaptation, et de la finalité de l'adaptation (Lalou, 2015, p.353). Pour Dounias et al., (2013, p.177), c'est la capacité d'un groupe d'acteur donné à maintenir en l'état de résilience le système socio-écologique dans lequel il évolue. Ainsi, dans la sous-préfecture de Séguéla, l'irrégularité dans la distribution des pluies poussent les exploitants agricoles à modifier le début de l'activités de débroussaillages et la date d'application des intrants. Si par le passé, elles s'effectuaient au moment de l'enregistrement des premières pluies aux mois de janvier et février, ces dernières années c'est au mois de mars que les ces dernières interviennent avec un intérêt pour la culture du riz dans les bas-fonds. Cette adaptation est confirmée par les travaux de Attané et al., (2015, p.117) qui soulignent que dans la région de Niakhar en pays Sereer, suite au démarrage tardif et à la fin précoce des pluies, la période de 1961-1990 a été marquée par des changements majeurs dans les pratiques agricoles : assèchement climatique et abandon progressif de la variété de mil à cycle long de 110 à 120 jours (sanio); généralisation du semis à sec du mil dans les bassins arachidiers et mise en culture des bas-fonds.

De plus, le développement des stratégies d'adaptation aux fluctuations pluviométriques conduit les exploitants agricoles de notre zone d'étude à adopter un système agricole basé sur des associations culturales afin de prévenir les risques d'échec d'une des activités. L'exemple de l'association du mil souna et du mil sanio, avec l'alternance des poquets dans un même champs chez les paysans de la région de Niakhar l'illustre bien (Attané et al., 2015, p.117). Cette transformabilité dans la stratégie de survie ou de résilience au quotidien des agriculteurs se retrouve également élaborée par les Luhya de façon à assurer la production et maintenir la fertilité des sols sur des exploitations dépassant rarement une superficie d'un hectare sont remarquables. Ces systèmes reposent sur des associations culturales des principales céréales vivrières (maïs et sorgho) avec une gamme étendue de cultures (légumineuses, légumes patates douces, bananes), de plantes médicinales et d'arbres fruitiers (Dounias et al., 2013, p. 179). Par ailleurs, pour Kosmowski et al., (2015, p.371), ces changements de pratiques sont liés à la sécurité alimentaire des ménages. Un meilleur rendement et surtout une production qui arrive plutôt sont les principales raisons poussant les exploitants à adopter de nouvelles variétés. Ce faisant, en considérant les incertitudes et les risques agricoles, les exploitants se réfèrent en premier lieu à l'échelle temporelle qui est celle du cycle agricole et de la période de soudure pour justifier ces changements. L'échelle temporelle de la pluviométrie ne semble pas ou peu intervenir dans leur prise de décision.

\section{Conclusion}

Les observations scientifiques et les perceptions paysannes du changement climatique façonnées par son impact dans la sous-préfecture de Séguéla conduisent ces derniers à des réponses adaptatives locales. Sur 30 années d'observations, 17 années sont caractérisées par la sècheresse. D'une part, ces observations scientifiques sont perçues en fonction des impacts (sol pauvre en eau) et des indicateurs dans le paysages agricoles (apparition de puces rouges indiquant la période de semence). D'autre part, elles sont basées sur les logiques des croyances locales qui lient «certains mauvais comportements» à la qualité des précipitations. L'étude montre également que face à la vulnérabilité des espaces et des populations des réponses adaptives tout comme les innovations individuelles et collectives sont mobilisées ; utilisation de facteurs de production (produits phytosanitaires), la succession culturale adaptée, le développement de l'association culturale pour améliorer la résilience au changement climatique en protégeant l'environnement dans la sous-préfecture de Séguéla. 


\section{Références}

1. AHMADI N., BASTIEN C., et TROMMETTER M., (2013), Observations, innovations et adaptations au changement climatique In S'adapter au changement climatique, Agriculture, écosystèmes et territoires (Soussana J-F., eds), Quae, Versailles, pp.61-73.

2. ATTANÉ A., OUMAROU A., et SOUMARÉ M. A., (2015), Quand la nature nous parle : une analyse comparée des représentations des changements climatiques et environnementaux In Les sociétés rurales face aux changements climatiques et environnementaux en Afrique de l'Ouest (Sultan B., Lalou R., Sanni M. A., Oumarou A., et Soumaré M. A., eds) IRD, Marseilles, pp.111110.

3. BROU Y. T., et CHALEARD J.L., (2007), Visions paysannes et changements environnementaux en Côte d'Ivoire, Annales de géographie, 2007/1 n653, pp.65-87.

4. DEME A., GAYE T. A., HOURDIN F., (2015), Les objectifs du climat en Afrique de l'Ouest : évidences et incertitudes In Les sociétés rurales face aux changements climatiques et environnementaux en Afrique de 1'Ouest (Sultan B., Lalou R., Sanni M. A., Oumarou A., et Soumaré M. A., eds) IRD, Marseilles, pp.61-87.

5. DOUNIAS E., VISSCHER de M-N., ICKOWICZ A., et CLOUVEL P., (2013), Les sociétés à agriculture de subsistance In S'adapter au changement climatique, Agriculture, écosystèmes et territoires (Soussana J-F., eds), Quae, Versailles, pp.171-194.

6. DUBREUIL V., ARVOR D., FUNATSU B., NÉDÉLEC V., et MELLO-THÉRY de N., (2021), Les changements climatiques en Amazonie, une approche multiscalaire In Les impacts spatiaux du changement climatique (Mercier D., eds) ISTE, London, pp.247-270

7. HANSEN J., MARX S., WEBER E., (2004), The role of climate perceptions, expectations and forecasts in farmer decision making: the Argentine Pampas and South Florida, Final Report of an IRI Seed Grant Project, 142p.

8. JOFFE H., (2005) De la perception à la représentation du risque: le rôle des médias CNRS, Hermès, 2005/1 n 41, pp.121-129

9. KOSMOWSKI F., GIBIGAYE M., MULLER B., et LALOU R., (2015), Innover en milieu rural Ouest africain : quels changements dans les pratiques agricoles des exploitants ? In Les sociétés rurales face aux changements climatiques et environnementaux en Afrique de l'Ouest (Sultan B., Lalou R., Sanni M. A., Oumarou A., et Soumaré M. A., eds) IRD, Marseilles, pp.359-375.

10. KOSMOWSKI F., LALOU R., SULTAN B., NDIAYE O., MULLER B., GALLE S., et SEGUIS L., (2015), Observations et perception des changements climatiques : Analyse comparée dans trois pays d'Afrique de l'Ouest In Les sociétés rurales face aux changements climatiques et environnementaux en Afrique de l'Ouest (Sultan B., Lalou R., Sanni M. A., Oumarou A., et Soumaré M. A., eds) IRD, Marseilles, pp.89-110.

11. L'HÔTE Y., MAHÉ G., SOMÉ B., et TRIBOULET J. P., (2002) Analysis of a Sahelian annual rainfall index from 1896 to 2000; the drought continues, Hydrological Sciences Journal, 47:4, pp.563-572.

12. LACLAU J-P., GAY F., BOUILLET J-P., BOUVET J-M., CHAIX G., DEMANGE A. C., DO F., EPRON D., FAVREAU B., GION J-M., NOUVELLON Y., PUJADE-RENAUD V., THALER P., VERHAEGEN D., VIGNERON P., (2015), Adaptation au changement climatique et atténuation dans les plantations d'arbres tropicaux In Changement climatique et agriculture du monde (Torquebiau E., eds), Quae, Versailles, pp.185-195.

13. LALOU R., (2015), Introduction In Les sociétés rurales face aux changements climatiques et environnementaux en Afrique de l'Ouest (Sultan B., Lalou R., Sanni M. A., Oumarou A., et Soumaré M. A., eds) IRD, Marseilles, pp.351-358. 